\title{
INSTABILITY OF A MAGNETIC FLUID DROP IN A CAPILLARY: A NUMERICAL STUDY
}

\author{
O. Lavrova ${ }^{1}$, V.Polevikov ${ }^{1}$, L. Tobiska ${ }^{2}$ \\ ${ }^{1}$ Belarusian State University, 4 Nezavisimosti Ave., Minsk, 220030 Belarus \\ ${ }^{2}$ Otto-von-Guericke University, Postfach 4120, D-39016 Magdeburg, Germany
}

In a magnetic field longitudinal to a cylindrical capillary, a magnetic fluid drop elongates until surface instability occurs. Two different types of instabilities arise as the magnetic field intensity increases. For fluids with small contact angles, the instability is caused by the drop breakage along the capillary axis with a subsequent fluid spreading over the capillary wall. For fluids with large contact angles, the instability is due to fluid separation from the capillary wall with a formation of a freely suspended drop inside the capillary. The main objective of the present work is a numerical study of the instability phenomena under a longitudinal uniform magnetic field. The problem is considered in a wide range of contact angles.

Introduction. Detailed investigations of the processes of magnetic fluid displacement in a capillary are necessary for the development of devices operating on the base of capillary-porous systems.

An applied magnetic field causes a magnetic fluid deformation in a capillary, see [1-4]. In a magnetic field longitudinal to the capillary, a magnetic fluid drop elongates until a surface instability occurs. Approximating the fluid surfaces by ellipsoids, two types of surface instabilities have been predicted in [1]. In the case of full wetting (contact angle equals $0^{\circ}$ ), the fluid drop breaks along the capillary axis and spreads over the capillary walls, when the field intensity reaches a critical value. For non-wetting fluids (contact angle equals $180^{\circ}$ ), the drop elongates in the central part of the capillary up to its separation from the wall. The surface instability in the capillary for non-wetting magnetic fluids was confirmed experimentally in [1], showing a good agreement with the theory. Numerical investigations of the instability phenomenon were presented in [4] under the assumption that the magnetic fluid is in a gap between two plates. A critical contact angle of $\alpha^{*}=117^{\circ}$ has been found, which separates the two types of surface instabilities. For $\alpha<\alpha^{*}$ the first type of instability occurs, whereas for $\alpha>\alpha^{*}$ the second one.

Here the influence of an external uniform magnetic field on the free surface shape of the magnetic fluid drop inside a cylindrical capillary is studied solving the coupled system of differential equations numerically. The problem is treated for magnetic fluids within a wide range of contact angles.

1. Mathematical modeling. Let a magnetic fluid of finite volume $V$ fill a cylindrical capillary of radius $a$. The fluid is hold by capillary forces over the capillary wall and wets it at a given contact angle $\alpha$. The capillary is placed in a uniform magnetic field of intensity $H_{0}$, directed along the capillary axis, under zero-gravity conditions. 
In the steady state the force balance is mathematically governed by a coupled system of Maxwell equations and Young-Laplace equation, see [5]

$$
\begin{aligned}
& \operatorname{rot} \mathbf{H}=\mathbf{0}, \quad \operatorname{div}(\mathbf{H}+\mathbf{M})=0 \quad \text { in } \Omega_{F} \cup \Omega_{A}, \\
& \sigma \mathcal{K}=\mu_{0} \int_{0}^{H} M \mathrm{~d} H+\frac{\mu_{0}}{2}(\mathbf{M} \cdot \mathbf{n})^{2}+C \quad \text { on } \Gamma .
\end{aligned}
$$

The Maxwell equations describe the structure of the magnetic field inside the magnetic fluid $\Omega_{F}$ and in the surrounding air $\Omega_{A}$. The Young-Laplace equation presents a balance of capillary and magnetic forces at the interface $\Gamma$ between fluid and air. Here, $\mathbf{H}$ denotes the magnetic field strength, $\mathbf{M}$ is the magnetization, $\sigma$ is the surface tension coefficient, $\mathcal{K}$ is the sum of principal curvatures, $\mu_{0}$ is the permeability in vacuum, $\mathbf{n}$ is the outward unit normal vector with respect to $\Omega_{F}$, and $C$ denotes a constant reference pressure. The magnetization of the fluid follows the Langevin law.

The fluid domain $\Omega_{F}$ has the form of a cylindrical body bounded by the free surfaces $\Gamma$ from the top and bottom. Due to symmetry of the fluid configuration with respect to the capillary axis, we reformulate the three-dimensional problem as a two-dimensional one in the meridional cross-section. Thus, only axisymmetric perturbations of the magnetic fluid are taken into consideration.

Using dimensionless variables, the physical parameters - the fluid volume $V$, the capillary radius $a$, the contact angle $\alpha$, the applied field intensity $H_{0}$, the initial susceptibility of the fluid $\chi$, the saturation magnetization $M_{\mathrm{S}}$, the permeability in vacuum $\mu_{0}$, and the surface tension coefficient $\sigma$ - are restricted to five dimensionless parameters: $U=V /\left(2 \pi a^{3}\right), \alpha, \chi, \gamma=3 \chi H_{0} / M_{\mathrm{S}}$ and $W=\mu_{0} M_{\mathrm{S}}^{2} a /(2 \sigma)$. The capillar radius $a$ and the field intensity $H_{0}$ are chosen as characteristic scales.

2. Numerical treatment. Problem (1) is formulated as a system of coupled equations. The magnetic fluid configuration and the free surface position as unknown quantities of the problem statement should be found simultaneously due to their interconnection. The free surfaces build domains $\Omega_{F}$ and $\Omega_{A}$ in the Maxwell equations, whereas free surfaces themselves are formed by magnetic forces in their surroundings. For the numerical treatment of the coupled equations (1), we apply a solution strategy based on a splitting to two subproblems: solving the Maxwell equations for a given interface position $\Gamma$ between fluid and air and calculating an equilibrium fluid shape from the given forces on the free surfaces. For a detailed description of the numerical solution strategy we refer to [6]. It has already been successfully applied to study plane capillary [4], Rosensweig instability $[7,8]$ and soliton-like surface configurations on the magnetic fluid layer $[9]$, recently discovered in [10].

The Maxwell equations are reformulated in terms of a potential $\varphi$, where $\mathbf{H}=\operatorname{grad} \varphi$. A dimensionless axisymmetric problem for the potential is defined in the cylindrical coordinates $r, z$ with a symmetry axis, directed parallel to the applied magnetic field. It results in a nonlinear elliptic equation inside the fluid meridian domain $\Omega_{F}$ and the Laplace equation in the air meridian domain $\Omega_{A}$ coupled by the continuity conditions for $\varphi$ and $\partial \varphi / \partial n$

$$
\begin{gathered}
\frac{\partial}{\partial r}\left(r \mu\left(\left|\operatorname{grad} \varphi^{(1)}\right|\right) \frac{\partial \varphi^{(1)}}{\partial r}\right)+\frac{\partial}{\partial z}\left(r \mu\left(\left|\operatorname{grad} \varphi^{(1)}\right|\right) \frac{\partial \varphi^{(1)}}{\partial z}\right)=0 \quad \text { in } \Omega_{F} \\
\frac{\partial}{\partial r}\left(r \frac{\partial \varphi^{(2)}}{\partial r}\right)+\frac{\partial}{\partial z}\left(r \frac{\partial \varphi^{(2)}}{\partial z}\right)=0 \quad \text { in } \Omega_{A}
\end{gathered}
$$




$$
\varphi^{(1)}=\varphi^{(2)}, \quad \mu \frac{\partial \varphi^{(1)}}{\partial n}=\frac{\partial \varphi^{(2)}}{\partial n} \quad \text { on } \partial \Omega_{F} ;
$$

where upper indices (1) and (2) denote variables in the fluid and in the air domains, respectively; $\partial \Omega_{F}$ is formed by the fluid-air interface $\Gamma$ and the capillary wall;

$$
\mu(h)=1+3 \chi(\operatorname{coth}(\gamma h)-1 /(\gamma h)) /(\gamma h), \quad h=\left|\operatorname{grad} \varphi^{(1)}\right| .
$$

The potential of the undisturbed uniform magnetic field is defined at infinity

$$
\lim _{(r, z) \rightarrow \infty}\left(\varphi^{(2)}-z\right)=0
$$

Additionally, the symmetry condition with respect to the $z$-axis is specified

$$
\frac{\partial \varphi^{(1)}}{\partial r}=0, \quad \frac{\partial \varphi^{(2)}}{\partial r}=0 \quad \text { for } r=0
$$

Problem (2)-(6) is solved numerically by a coupled finite element and boundary element method elaborated in the cylindrical coordinates especially for the considered problem (see [11]). The finite element discretization is applied for the nonlinear elliptic equation to find a piecewise-linear approximation for $\varphi^{(1)}$ in $\Omega_{F}$ and a piecewise-constant approximation for $\partial \varphi^{(1)} / \partial n=q^{(1)}$ on $\partial \Omega_{F}$

$$
\int_{\Omega_{F}} \mu(h)\left(\frac{\partial \varphi^{(1)}}{\partial r} \frac{\partial v}{\partial r}+\frac{\partial \varphi^{(1)}}{\partial z} \frac{\partial v}{\partial z}\right) r \mathrm{~d} r \mathrm{~d} z=\int_{\partial \Omega_{F}} \mu(h) q^{(1)} v r \mathrm{~d} s
$$

for all piecewise-linear functions $v(r, z)$ defined on a triangulation of $\Omega_{F}$. Fig. 2 presents Delaunay triangulations of $\Omega_{F}$ constructed by means of the software package Triangle, see [12]. The boundary element technique applied for the Laplace equation in $\Omega_{A}$ with the condition at infinity (5) and further application of the continuity conditions (4) results in the integral equation for $\varphi^{(1)}$ and $q^{(1)}$ on $\partial \Omega_{F}$

$$
\frac{\varphi^{(1)}\left(\xi^{0}\right)}{2}-\int_{\partial \Omega_{F}} q_{a x}^{*}\left(\xi^{0}, \xi\right) \varphi^{(1)}(s) r(s) \mathrm{d} s=z^{0}-\int_{\partial \Omega_{F}} u_{a x}^{*}\left(\xi^{0}, \xi\right) \mu(h) q^{(1)}(s) r(s) \mathrm{d} s .
$$

Here, $\xi^{0}=\left(r^{0}, z^{0}\right) \in \partial \Omega_{F}, \xi=(r(s), z(s)) \in \partial \Omega_{F} ; u_{a x}^{*}\left(\xi^{0}, \xi\right)=K(m) /(\pi \sqrt{a+b})$,

$$
\begin{gathered}
q_{a x}^{*}\left(\xi^{0}, \xi\right)=\frac{1}{\pi \sqrt{a+b}}\left\{-\frac{z^{\prime}}{2 r}[E(m)-K(m)]+\frac{z^{\prime}\left(r-r^{0}\right)-r^{\prime}\left(z-z^{0}\right)}{\left|\xi-\xi^{0}\right|^{2}} E(m)\right\} ; \\
K(m)=\int_{0}^{\pi / 2} \frac{\mathrm{d} \theta}{\sqrt{1-m \sin ^{2} \theta}}, \quad E(m)=\int_{0}^{\pi / 2} \sqrt{1-m \sin ^{2} \theta \mathrm{d} \theta} ; \quad m=\frac{2 b}{a+b} \leq 1 ; \\
a=\left|\xi-\xi^{0}\right|^{2}+b, \quad b=2 r^{0} r ; \quad r=r(s), \quad z=z(s) ;
\end{gathered}
$$

$K(m)$ and $E(m)$ are the complete elliptic integrals of the first and second kind. We point out that $K(m) \rightarrow+\infty$ for $m \rightarrow 1$. A collocation technique is applied for the discretization of the integral equation (8) with collocation points $\xi^{0}$, lying in the middle of every boundary element. A coupled boundary and finite element discretization of equations (7)-(8) results in a set of nonlinear equations for $\varphi^{(1)}$ in $\Omega_{F}$ and $q^{(1)}$ on $\partial \Omega_{F}$. The nonlinear system is linearized by the fixedpoint method. The Gaussian elimination method is a suitable approach to solve linearized systems. 
In contrast to the plane case, the boundary integrals in (8) do not allow exact calculations. For the case $\xi \neq \xi^{0}$, Gaussian quadratures are applied. For the case $\xi=\xi^{0}$, the integrals are singular because $K(m) \rightarrow \infty$. To resolve singularities, an approximation for $K(m)$ is used, see [13]

$$
K(m)=\sum_{k=0}^{4}\left[\alpha_{k} m_{1}^{k}+\beta_{k} m_{1}^{k} \ln \left(1 / m_{1}\right)\right] ; \quad m_{1}=1-m .
$$

As a result, the integrals have only logarithmic singularities, which could be accurately handled by logarithmically weighted Gaussian quadratures.

The free surface is parameterized along the arc length $s, \Gamma=\{r=R(s), z=$ $Z(s), s \in[0, L]\}$, resulting in the reformulation of the Young-Laplace equation as a second order system of ordinary differential equations for the unknown functions $R(s)$ and $Z(s)$ together with two unknown parameters $C$ and $L$, see [14]. A finite difference scheme of the second order, see [14], is used for the approximation, which has been sucessfully applied to a variety of ferrohydrostatic problems $[4,7$, $9,11,14]$. The additional use of an adaptive grid construction, see [14], increases significantly the accuracy of the computations.

3. Results of numerical simulations. Two types of surface instabilities in the cylindrical capillary are numerically detected. The first type of instability (fluid breakage along the capillary axis and spreading over the wall) is numerically predicted for contact angles $\alpha<\alpha^{*}$, whereas the second type of instability (fluid separation from the capillary wall) takes place for $\alpha>\alpha^{*}$. The critical contact angle $\alpha^{*}$, separating the two types of instabilities, slightly depends on the fluid volume: $\alpha^{*}=117.7^{\circ}$ for $U=1, \alpha^{*}=121.3^{\circ}$ for $U=2, \alpha^{*}=123.8^{\circ}$ for $U=4$, and is close to the numerically found value $\alpha^{*}=117^{\circ}$ for the plane capillary in [4].

Fig. 1 shows the critical values of the applied magnetic field $\gamma^{*}(\alpha)$, at which the surface instability is numerically predicted. A stronger magnetic field is required to

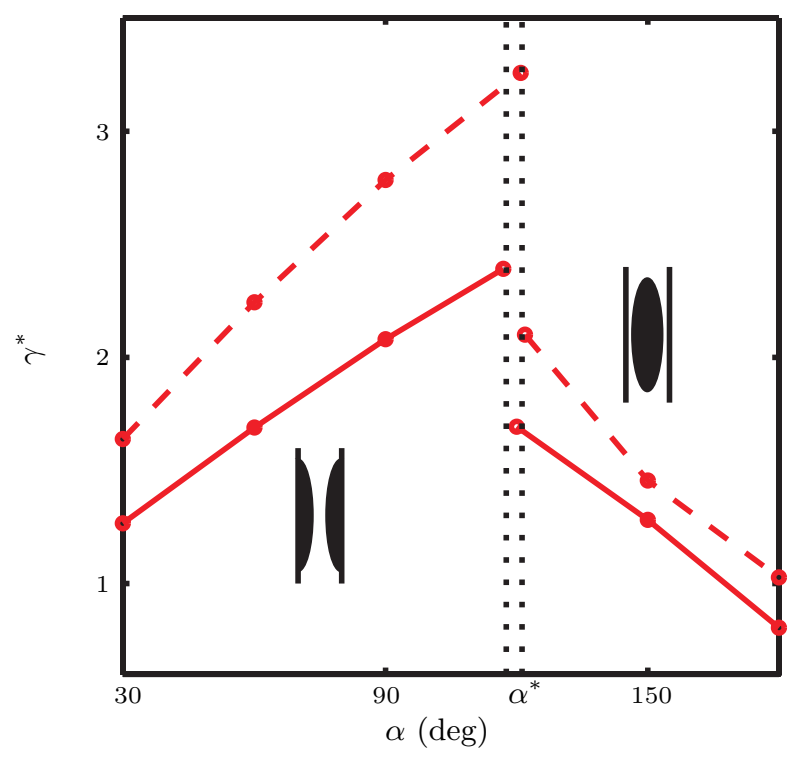

Fig. 1. Critical value of the applied magnetic filed versus the contact angle: $U=1$ (solid line), $U=2$ (dashed line), $\chi=5, W=100$. Critical angle $\alpha^{*}$ separates two types of instabilities. 


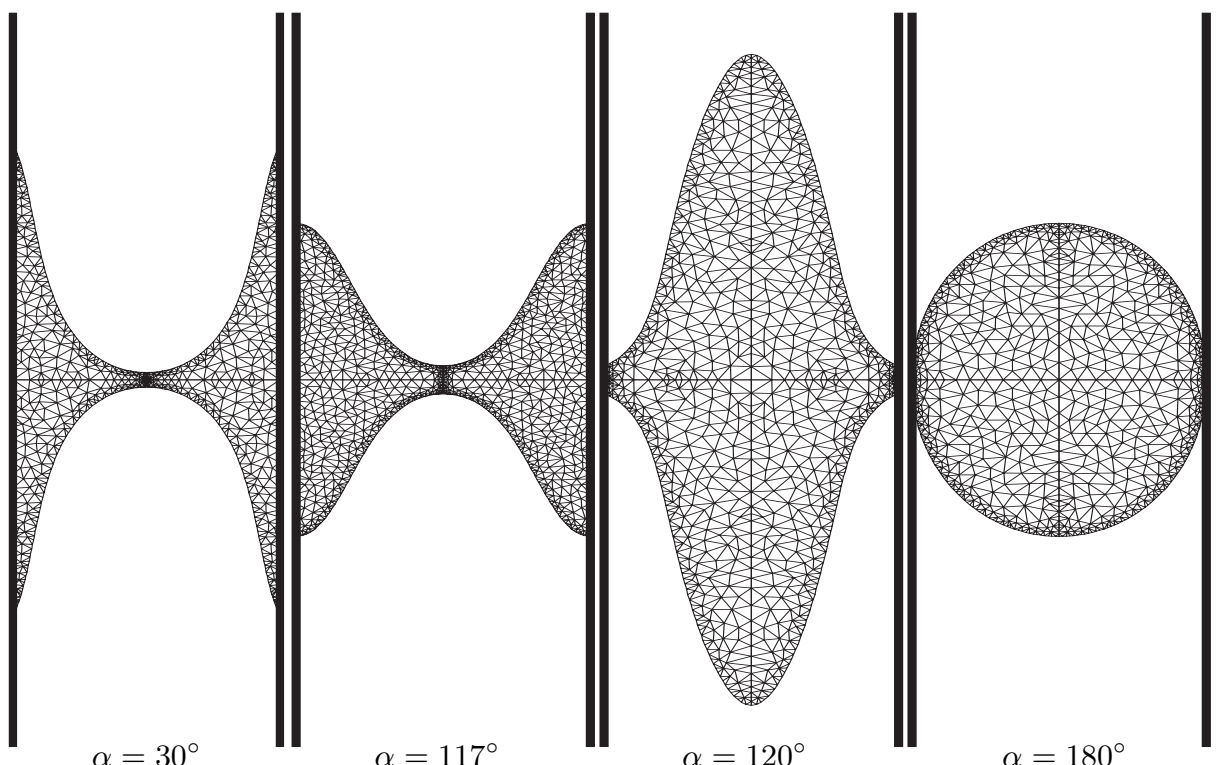

Fig. 2. Axial section of the capillary at $\gamma=\gamma^{*}: U=1, \chi=5, W=100$.

reach instability for contact angles close to $\alpha^{*}$, whereas for $\alpha \rightarrow 0^{\circ}$ and $\alpha \rightarrow 180^{\circ}$ the value of $\gamma^{*}$ is monotonically decreasing.

Fig. 2 shows axial sections of the capillary with equilibrium drop configurations at the critical magnetic fields $\gamma=\gamma^{*}$. For all pictures in Fig. 2 the magnetic fluid occupies an equal volume $U=1$, showing nevertheless different areas in axial sections. As equilibrium states for $\gamma>\gamma^{*}$, we expect double-connected fluid domains when the drop thickness over the capillary axis tends to zero (see Fig. 2 for $\alpha=30^{\circ}$ and $117^{\circ}$ ) or expect isolated drops when the fluid separates from the capillary wall (see Fig. 2 for $\alpha=120^{\circ}$ and $180^{\circ}$ ). Such surface configurations are excluded from the model statement due to the assumption that the free surface $\Gamma$
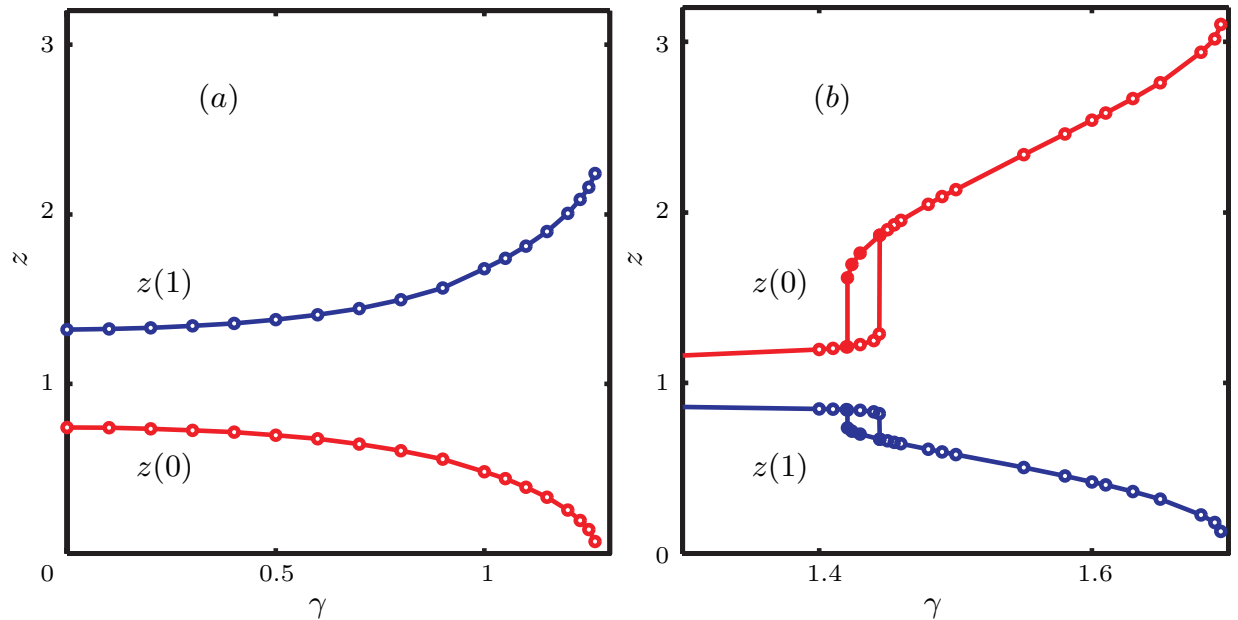

Fig. 3. Drop thickness over the capillary axis $z(0)$ and the capillary wall $z(1)$ versus the applied field: $U=1, \chi=5, W=100, \alpha=30^{\circ}(a), \alpha=120^{\circ}(b)$. Empty (full) markers display results for increasing (decreasing) field. 
touches the capillary wall crossing at the same time the capillary axis. As a result, numerical simulations are limited by intensities $\gamma<\gamma^{*}(\alpha)$. It is found numerically that for the increasing intensity of the applied field the drop thickness over the capillary axis $\left(\alpha<\alpha^{*}\right)$ or over the capillary wall $\left(\alpha>\alpha^{*}\right)$ tends to zero value, indicating in such a way onset of the surface instability, see, e.g., Fig. 2 or Fig. 3.

Fig. 3 presents the drop elongation in the capillary versus the applied magnetic field. The fluid thickness over the capillary axis $z(0)$ and the capillary wall $z(1)$ are shown for $\alpha=30^{\circ}$ (first type of instability) and for $\alpha=120^{\circ}$ (second type of instability).

For $\alpha=30^{\circ}$, the free surface is concave at zero field $(z(0)<z(1))$ and formed by an elliptic curve. Concave configurations of the free surface are preserved for increasing fields up to $\gamma=\gamma^{*}$, where the surface instability appears. The drop thickness over the capillary axis $z(0)$ monotonically decreases to zero, whereas the wetting area of the capillary wall $z(1)$ monotonically increases. A similar behaviour is shown for fluids with $\alpha \leq 90^{\circ}<\alpha^{*}$. The shape of magnetic fluids with $90^{\circ}<\alpha<\alpha^{*}$ is convex $(z(0)>z(1))$ at zero field. A monotonous decrease of $z(0)$ and an increase of $z(1)$ with increasing fields shows a transition from convex to concave surface configurations and results finally in the first type of instability.

For $\alpha=120^{\circ}$ (Fig. 3b), the drop elongates over the capillary axis $(z(0)$ increases) up to its separation from the wall $(z(1)$ decreases to zero). All fluids with $\alpha>\alpha^{*}$ behave analogously. It is observed numerically that for fluids with $\alpha>\alpha^{*}$ and close to $\alpha^{*}$ the drop elongation shows a hysteretic behaviour (see Fig. $3 b$ ). Let us increase the field intensity starting from zero. The free surface keeps nearly unchangeable with $z(0) / z(1) \approx 1.4$ up to some threshold $\bar{\gamma}=1.4443$, at which the drop jumps to a more elongated configuration with $z(0) / z(1) \approx 2.8$. At $\bar{\gamma}<\gamma<\gamma^{*}$, the drop elongates over the capillary axis smoothly. For decreasing fields, the drop smoothly shrinks over the capillary axis up to $\gamma=1.4207<\bar{\gamma}$, where the drop jumps to a less elongated configuration. For fields in the region $\underline{\gamma}<\gamma<\bar{\gamma}$, two equilibrium surface configurations exist. We point out that a hysteretic behaviour of freely suspended drops was numerically detected in [11] for magnetic fluids with high susceptibilities $\chi \geq 21$.

\section{REFERENCES}

[1] V. Bashtovoi, P. Kuzhir and A. Reks. Capillary ascention of magnetic fluids. J. Magn. Magn. Mater., Vol. 252 (2002), pp. 265-267.

[2] V. Bashtovoi, P. KuzhiR, A. Reks, G. Bossis and O. Volkova. The uniform magnetic field influence on the magnetic fluid meniscus motion in the cylindrical capillary. Int. J. Mod. Phys. B, Vol. 16 (2002), pp. 2590-2596.

[3] V. Bashtovoi, G. Bossis, P. Kuzhir and A. Reks. Magnetic field effect on capillary rise of magnetic fluids. J. Magn. Magn. Mater., Vol. 289 (2005), pp. 376-378.

[4] V. Polevikov and L. Tobiska. Instability of magnetic fluid in a narrow gap between plates. J. Magn. Magn. Mater., Vol. 289 (2005), pp. 379-381.

[5] R. E. Rosensweig. Ferrohydrodynamics. (Cambridge University Press, New York, 1985).

[6] O. Lavrova, G. Matthies, T. Mitkova, V. Polevikov and L. Tobiska. Numerical treatment of free surface problems in ferrohydrodynamcs. J. Phys.: Condens. Matter, Vol. 18 (2006), pp. 2657-2669. 
[7] V. Bashtovoi, O. Lavrova, V. Polevikov and L. Tobiska. Computer modelling of the instability of a horizontal magnetic-fluid layer in a uniform magnetic field. J. Magn. Magn. Mater., Vol. 252 (2002), pp. 299-301.

[8] G. Matthies and L. TobiskA. Numerical simulation of normal-field instability in the static and dynamic case. J. Magn. Magn. Mater., Vol. 289 (2005), pp. 346-349.

[9] O. Lavrova, G. Matthies and L. Tobiska. Numerical study of solitonlike surface configurations on a magnetic fluid layer in the Rosensweig instability. Commun. Nonlinear Sci. Numer. Simul. Vol. 13 (2008), pp. 1302-1310.

[10] R. Richter And I. Barashenkov. Two-dimensional solitons on the surface of magnetic fluids. Phys. Rev. Lett, Vol. 94 (2005), pp. 184503-184506.

[11] O. Lavrova. Numerical methods for axisymmetric equilibrium magneticfluidshapes. Ph.D. thesis, Otto-von-Guericke University Magdeburg, Germany, 2006.

[12] J.R. ShewChuK. Triangle: engineering a 2D quality mesh generator and Delaunay triangulator. In: Lecture Notes in Computer Science. (SpringerVerlag, Berlin, 1996), Vol. 1148, pp. 203-222.

[13] M. Abramowitz And I. A. Stegun. Handbook of Mathematical Functions. (Dover, New York, 1965).

[14] V. Polevikov. Methods for numerical modeling of two-dimensional capillary surfaces. Comput. Methods in Applied Math., Vol. 4 (2004), pp. 66-93.

Received 02.04.2008 